\title{
Fatores críticos para a melhoria contínua em indústrias brasileiras
}

\author{
Pedro Carlos Oprime, ${ }^{\mathrm{a}, *}$, Glauco Henrique de Sousa Mendes ${ }^{\mathrm{b}}$, \\ Márcio Lopes Pimenta ${ }^{\mathrm{c}}$ \\ a,*pedro@dep.ufscar.br, UFSCar, Brasil \\ bglauco@dep.ufscar.br, UFSCar, Brasil \\ cpimenta@unifei.edu.br, UNIFEl, Brasil
}

\begin{abstract}
Resumo
0 objetivo deste artigo é identificar e analisar fatores críticos no desenvolvimento de atividades de melhoria contínua (MC) em empresas industriais brasileiras. Um modelo conceitual de relacionamento entre práticas e resultados foi testado por meio de um survey utilizando uma amostra de 46 empresas industriais. Fatores como: treinamento em ferramentas de solução de problemas, o incentivo a sugestões, a comunicação face a face, visitas ao chão de fábrica e adoção de sistemas de incentivos, se mostraram críticos para alcance de sucesso em atividades de MC. Identificaram pela análise fatorial dois constructos críticos relacionados ao processo de melhoria contínua: 1) a promoção das atividades de melhoria contínua por meio de políticas de incentivos; e 2) o suporte e liderança da alta administração e ativa participação da gerência. Observou-se que há relação estatisticamente significativa entre o uso de técnicas de solução de problemas e mecanismos de incentivos com o desempenho das empresas.
\end{abstract}

Palavras-chave

Melhoria contínua. Fatores críticos. Análise fatorial.

\section{Introdução}

A Melhoria Contínua (MC) pode ser considerada como um dos fundamentos dos sistemas de produção baseados nos modelos de gestão da qualidade total, produção enxuta e manufatura de classe mundial. Embora frequentemente seja associada a tais modelos, a MC pode ser implementada como um programa independente, que produz avanços cumulativos nos indicadores de resultados de uma organização. Nesse sentido, a MC é uma importante ferramenta estratégica para aumentar a competitividade de qualquer organização (MARIN-GARCIA; VAL; MARTIN, 2008).

Caffiyn (1999) conceitua MC como um processo concentrado na inovação incremental que deve envolver toda a organização. Segundo Bessant e Francis (1999), a MC é resultado de três elementos: caminhos, posição e processo. Os dois primeiros estão relacionados ao conjunto de competências que uma organização acumula ao longo do tempo e ao posicionamento em termos de produto/mercado que pretende adotar. 0 terceiro elemento diz respeito às rotinas da organização que determinam sua forma de inovar, aprender e renovar.

Muitos temas têm sido relacionados à MC. Entre eles, merecem destaque os modelos de organização das equipes de melhoria (AUGSDORFER; HARDING, 1995; DABHILKAR; BENGTSSON, 2004); a formação de habilidades dos membros das equipes de melhoria (BESSANT; FRANCIS, 1999; JABNOUN, 2001; LEE, 2004; ABRAHAMSSON; GERDIN, 2006); o relacionamento entre $\mathrm{MC}$ e sistemas de gestão da qualidade (CHAPMAN; HYLAND, 1997; HYLAND et al., 2000); a utilização de mecanismos de suporte e ferramentas (DELBRIDGE; BARTON, 2002); o uso de indicadores de desempenho e sistemas de informação como suporte às atividades de $M C$ (BECHET; WAINWRIGHT; BANCE, 2000).

A identificação de fatores críticos que positivamente influenciam o resultado das ações de MC tem sido estudada por Atkinson (1994) e 
Marin-Garcia, Val e Martin (2008). Entretanto, muitas organizações têm encontrado dificuldades para, de forma efetiva, implementar esse conceito.

A bibliografia desse tema sugere que mais pesquisas empíricas devem ser conduzidas para identificar os fatores que realmente contribuem para o sucesso das atividades de MC e, consequentemente, para o próprio desempenho da organização. A proposta deste artigo é:

- Analisar o impacto dos meios e técnicas utilizadas no apoio e suporte à identificação de problemas nos resultados das atividades de MC;

- Analisar a influência dos sistemas de incentivos nos resultados das atividades de MC;

- Identificar as ferramentas que mais contribuem para o sucesso das atividades de MC; e

- Analisar a relação entre as práticas de operacionalização das atividades de MC e os resultados financeiros e não financeiros.

Para cumprir este propósito foi realizado um levantamento (survey) em empresas brasileiras que adotam sistemas formais de gestão da qualidade. Os dados coletados foram analisados por meio de técnicas estatísticas descritivas e multivariadas.

As seções seguintes apresentam uma revisão sobre MC, seguida da formulação das hipóteses da pesquisa, bem como os procedimentos metodológicos incluindo métodos não paramétricos e análise fatorial. Por fim, as considerações finais apresentam os fatores críticos obtidos no levantamento.

\section{Melhoria contínua}

Savolainen (1999) afirma que a implementação de ações de Melhoria Contínua pode ser considerada como um processo de renovação organizacional a partir da introdução de novos comportamentos e reformas na estrutura administrativa, em especial na ideologia e nas práticas gerenciais.

A MC consiste em um amplo processo organizacional focado e sustentado por uma abordagem orientada à melhoria incremental. É considerada uma importante ferramenta gerencial para que a organização molde sua estratégia competitiva frente às turbulências e incertezas do ambiente externo (BESSANT; FRANCIS, 1999).

A ideia de MC está relacionada à capacidade de resolução de problemas por meio de pequenos passos, alta frequência e ciclos curtos de mudança (BESSANT et al., 1994). De maneira semelhante, Boer et al. (2000) descrevem MC como um processo planejado, organizado e sistemático de realização de mudanças incrementais nas práticas existentes na organização com consequências no desempenho organizacional.

Bessant et al. (1994) e Hyland et al. (2000) enfatizam aspectos comportamentais em suas definições de MC. Caffyn (1999) aponta a MC como a habilidade da empresa em superar seus competidores por meio da inovação e do envolvimento dos empregados. Essas definições demonstram a necessidade de envolvimento do maior número possível de pessoas na organização, as quais são chamadas a contribuir com pequenas melhorias em produtos e processos, assim como a compartilharem suas experiências, conhecimentos e aprendizados (BESSANT; CAFFYN, 1997).

Bessante Caffyn (1997) identificam competências fundamentais associadas à MC. Essas competências representam os padrões de comportamentos que devem estar presentes na organização e são definidas por: i) habilidade organizacional (capacidade ou aptidão para adotar uma abordagem particular para melhoria contínua; ii) comportamento constitutivo (rotinas de comportamento que os funcionários adotam e que reforçam a abordagem da MC) e iii) facilitadores (procedimentos e técnicas usadas para avançar o esforço de MC).

Podem ser identificados diferentes níveis de maturidade na implementação de programas de MC. Bessant, Caffyn e Gallagher (2001) apontam cinco estágios de evolução da prática de $\mathrm{MC}$ nas organizações. Esses estágios vão de 1 a 5, sendo o primeiro (pré-melhoria) quando a organização introduz o conceito de $M C$ e esta não influencia o desempenho organizacional. No quinto nível (capacidade total de MC), toda a organização está envolvida em atividades de melhoria focadas em inovações incrementais e radicais. Também ocorre o compartilhamento de conhecimentos e experiências, aproximando-a do modelo de aprendizagem organizacional.

Os estágios devem funcionar como guias de orientação para que a organização possa definir estratégias a fim de incrementar suas capacidades e alcançar níveis superiores de maturidade (ATTADIA; MARTINS, 2003). Trata-se de um processo estratégico que precisa ser administrado com foco no longo prazo (BESSANT et al., 1994).

Diante deste conteúdo que faz introdução ao conceito de MC, cabe discutir, a seguir, questões importantes para que as atividades de melhoria sejam eficiente e eficazmente implementadas.

\subsection{Fatores críticos nas atividades de melhoria contínua}

Diversos estudos (ATKINSON, 1994; CHAPMAN; HYLAND, 1997; BECHET; WAINWRIGHT; BANCE, 
2000; BOND, 1999; HARRISON, 2000; JABNOUN, 2001; HYLAND; SOOSAY; SLOAN, 2003; FAGER et al., 2004; LEE, 2004; FRYER; ANTONY; DOUGLAS, 2007; MARIN-GARCIA; VAL; MARTIN, 2008) identificam conjuntos de fatores que afetam as atividades e todo o processo de MC em uma organização, a saber:

- Os aspectos culturais e paradigmas vigentes;

- A organização dos grupos (organizado em força tarefa, em grupos semiautônomos, com cooperação e comunicação lateral, uso de facilitadores e especialistas em técnicas e métodos de solução de problemas e suporte gerencial);

- A governança dos grupos (centralizado ou descentralizado, através de rotinas e métodos especificados e acompanhamento das atividades);

- 0 domínio e aplicação de técnicas e ferramentas na identificação e solução de problemas; e

- 0 desenvolvimento de um sistema de informação para auxiliar planejamento e controle das atividades.

Os aspectos culturais e paradigmas vigentes na organização podem influenciar positiva ou negativamente em sua capacidade de adquirir e incorporar comportamentos essenciais para o sucesso do programa de MC. Um valor essencial é desenvolver a habilidade para a identificação e solução de problemas. lsso pode ser executado por meio da cooperação interna ou externa, que implica em comunicação clara e direta, confiança entre as partes e, muitas vezes, administração de conflitos (JABNOUN, 2001; LEE, 2004).

Fatores comportamentais do gerenciamento, tais como liderança e gerenciamento de pessoas também estão relacionados aos aspectos culturais, pois fazem parte de um processo social que envolve novas relações, papéis, modos de cooperação e estrutura de controle, que podem facilitar ou inibir as atividades de MC (HARRISON, 2000).

$\mathrm{Na}$ formação dos grupos de melhoria, Harrington (1995) afirma que a MC é um processo que se desenvolve de baixo para cima (bottom-up), enquanto a melhoria radical implementa-se de cima para baixo (top-down). Terziovski (2002) complementa ao ressaltar que os grupos bottom-up de melhoria incremental (típicos da abordagem Kaizen) são mais recomendados para a melhoria da produtividade e da satisfação dos clientes. Já os grupos top-down são mais apropriados para geração de inovações tecnológicas.

Os gerentes têm importante papel no processo de implantação da MC, uma vez que atuam em sua governança por meio da adoção de rotinas e metas definidas para cada grupo. De um lado, o formalismo excessivo e centralizador inibe a participação dos indivíduos; de outro lado, a liberdade excessiva gera resultados instáveis pela falta de comprometimento da gerência (DABHILKAR; BENGTSSON, 2004).

Por isso é recomendado o balanceamento entre o uso de mecanismos de controle associado ao envolvimento das pessoas. Essa postura gerencial facilita a comunicação e cooperação lateral, a autodeterminação dos indivíduos e grupos, ao mesmo tempo em que gera estabilidade ao programa de melhoria. Esse aspecto insere-se nas teorias sobre mudanças culturais e sistemas de ideias apresentadas por vários autores (BESSANT; FRANCIS, 1999; SAVOLAINEN, 1999; MURRAY; CHAPMAN, 2003; DABHILKAR; BENGTSSON, 2004; ABRAHAMSSON; GERDIN, 2006).

Terziovsky e Sohal (2000) identificaram as ferramentas mais comumente utilizadas por empresas australianas do setor manufatureiro e os impactos dessas ferramentas no sucesso das atividades de MC. Os resultados evidenciaram a preferência quanto ao uso de técnicas para identificação e solução de problemas mais simples como checklists, as sete ferramentas básicas da qualidade e as ferramentas de mapeamento de processos. Não foram estatisticamente significativas as técnicas mais avançadas de solução de problemas (QFD - Quality Function Development e FMEA Failure Model and Effect Analysis) as técnicas soft (relacionadas a pessoas e grupos de melhoria).

Por fim, Bechet, Wainwright e Bance (2000) e Bond (1999) ressaltam a importância de sistemas de informação para facilitar a coleta e análise de dados de processo e produto, bem como gerar indicadores de desempenho que facilitem e apoiem as atividades dos grupos de melhoria. Do mesmo modo, Atkinson (1994) indica o uso de medidas de desempenho como fator-chave para a MC.

Diante desse complexo conjunto de elementos que influenciam o sucesso das atividades de MC, muitas organizações encontram dificuldades em implementá-la. Tal aspecto desperta o interesse do setor acadêmico para execução de investigações empíricas sobre esse fato. Nesse sentido, foi consultada a bibliografia sintetizada nesta revisão para propor um modelo conceitual que relacione práticas e resultados.

\subsection{Modelo conceitual}

A Figura 1 apresenta o modelo conceitual que direcionou a pesquisa apresentada no artigo. As variáveis individuais dependentes (práticas relacionadas à $\mathrm{MC}$ ) foram agrupadas em três grandes constructos: práticas de operacionalização, incentivos às atividades de $\mathrm{MC}$ e ferramentas de apoio às atividades de MC. 
Como se observa na Figura 1, a hipótese $\mathrm{H} 1$ sugere que práticas de operacionalização exercem influência positiva no desempenho das atividades de MC. Já as hipóteses $\mathrm{H} 2$ e H3 cogitam a existência de influência positiva dos sistemas de incentivo e das ferramentas de apoio operacional sobre desempenho das atividades de MC.

Para aprofundar o entendimento dos elementos contidos na Figura 1, o Quadro 1 apresenta as variáveis individuais pertencentes a cada constructo.

\section{Método de pesquisa}

A pesquisa foi projetada em quatro etapas. Primeiramente, realizou-se uma revisão biblio-

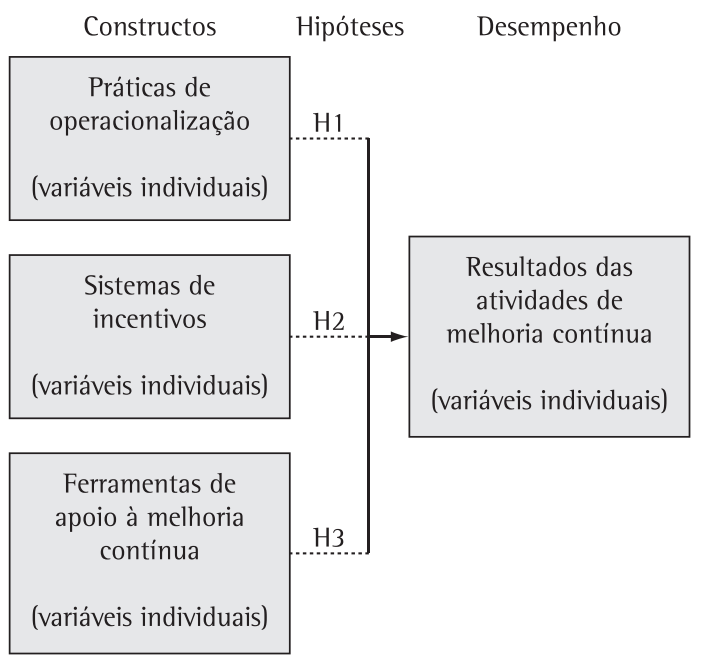

Figura 1. Modelo conceitual da pesquisa. gráfica, que permitiu a identificação dos fatores críticos na condução das atividades de MC. Os referidos fatores foram incorporados ao modelo conceitual proposto. $\mathrm{Na}$ segunda etapa, foi elaborado e testado o questionário da pesquisa. $\mathrm{Na}$ terceira, foi levada a efeito a coleta dos dados. $\mathrm{Na}$ quarta etapa, foram realizadas análises estatísticas dos dados coletados.

\subsection{Amostra de empresas}

A pesquisa foi conduzida por meio de um levantamento (survey). Inicialmente, e-mails foram enviados às empresas, convidando-as a participar da pesquisa e explicando-lhes os procedimentos de resposta. Foi utilizada a base de dados da ABNT (Associação Brasileira de Normas Técnicas), para identificar empresas com certificação ISO 9001. Houve a preocupação de direcionar o questionário para pessoas com responsabilidade ou participação direta na gestão dos programas ou atividades de melhoria. Se essa premissa não havia sido respeitada, os questionários eram desconsiderados. No total foram enviados e-mails a 1.221 empresas de diversos estados do Brasil.

Após esse primeiro procedimento, as empresas que haviam iniciado a resposta do questionário foram contatadas por telefone, para esclarecer possíveis dúvidas, e incentivadas a preencher o questionário por completo. Esses esforços resultaram uma amostra composta por 46 empresas, que, ao final de 2007, foi definida como a amostra possível levantada do universo identificado.

Quadro 1. Variáveis individuais investigadas na pesquisa.

\begin{tabular}{|c|c|c|c|}
\hline Práticas de operacionalização & Sistemas de incentivos & Ferramentas de apoio & $\begin{array}{l}\text { Resultados das } \\
\text { atividades de MC }\end{array}$ \\
\hline $\begin{array}{l}\text { Uso de slogan; } \\
\text { Treinamento de pessoal em ferramentas } \\
\text { de solução de problemas; } \\
\text { Monitoramento das atividades de melhoria } \\
\text { (medidas, acompanhamento); } \\
\text { Suporte do staff gerencial; } \\
\text { Sistemas de incentivo; } \\
\text { Liderança que dá suporte; } \\
\text { Trabalho em grupo; } \\
\text { Plano de sugestões; } \\
\text { Formato genérico de solução de } \\
\text { problemas; } \\
\text { Promoção no quadro de avisos; } \\
\text { Promoção por mídia interna; } \\
\text { Emprego de política formal; } \\
\text { Comunicação face a face; } \\
\text { Visitas regulares ao chão de fábrica pela } \\
\text { gerência; } \\
\text { Uso do ISO 9000/200o ou outros padrões } \\
\text { de qualidade; } \\
\text { Uso de manutenção preventiva total; } \\
\text { Prêmios da qualidade; e } \\
\text { Promoção por competição e prêmio. }\end{array}$ & $\begin{array}{l}\text { Sugestões são avaliadas e } \\
\text { recompensadas; } \\
\text { Resultados das melhorias } \\
\text { são recompensados } \\
\text { diretamente através de } \\
\text { bônus/prêmios; } \\
\text { Resultados das melhorias } \\
\text { são recompensados } \\
\text { indiretamente através de } \\
\text { salários individuais; } \\
\text { Sistemas de incentivo; e } \\
\text { Resultados das melhorias } \\
\text { são recompensados a todo } \\
\text { o time }\end{array}$ & $\begin{array}{l}\text { Ferramentas de identificação de } \\
\text { problemas/checklist; } \\
7 \text { ferramentas básicas da qualidades } \\
\text { ex: Pareto, diagrama de espinha de } \\
\text { peixe; } \\
7 \text { ferramentas “novas" da qualidade } \\
\text { (7 ferramentas gerenciais da qualidade) } \\
\text { ex: diagrama de afinidades; } \\
\text { Ferramentas de mapeamento de } \\
\text { processos; } \\
\text { FMEA (Análise dos Modos de Falha e } \\
\text { seus Efeitos); } \\
\text { QFD (Desdobramento da função } \\
\text { qualidade); } \\
\text { Ferramentas de criatividade/ } \\
\text { Ferramentas de visualização/ display, } \\
\text { Ferramentas de padronização; } \\
\text { 5S; } \\
\text { Simulação; } \\
\text { Seis Sigma; e } \\
\text { Controle Estatístico do Processo (CEP). }\end{array}$ & $\begin{array}{l}\text { Aumento da produtividade; } \\
\text { Melhoria na conformidade } \\
\text { da qualidade; } \\
\text { Redução do lead time; } \\
\text { Redução de custo; } \\
\text { Aumento da satisfação do } \\
\text { cliente; e } \\
\text { Aumento da habilidade dos } \\
\text { funcionários. }\end{array}$ \\
\hline
\end{tabular}


Seguindo a classificação de porte das empresas do SEBRAE (Serviço Brasileiro de Apoio às Micro e Pequenas Empresas), a amostra ficou com a seguinte composição: $44,44 \%$ de empresas de médio porte (de 100 a 499 empregados); 37,78\% de pequeno porte (de 20 a 99 empregados), $13,33 \%$ de grande porte (mais de 500 empregados) e 4,44\% de microempresas. Em relação aos setores da economia, diversos tipos de indústrias estão presentes na amostra, motivo pelo qual ela pode ser considerada como possuidora de relativo grau de representatividade do amplo espectro de setores que compõem o parque industrial brasileiro. Os setores presentes na amostra são: produtos metálicos $(27 \%)$, produtos químicos (18\%), eletrônicos e ópticos (16\%), borrachas e plásticos (11\%), máquinas e equipamentos (11\%), petróleo e equipamentos de transporte (7\%), papel e celulose ( $4 \%$ das empresas), fabricação de outros produtos minerais não metálicos $(2 \%)$ e outros setores (4\%).

\subsection{Instrumento de pesquisa}

Para coleta de dados utilizou-se um questionário estruturado que foi disponibilizado e respondido pela Internet. Antes de sua aplicação definitiva, ele foi aprimorado por meio de três pré-testes com especialistas da área (profissionais responsáveis pelas atividades e programas de MC em empresas com certificação 1SO 9001:2000) para garantir o entendimento apropriado dos significados dos termos utilizados, dos conteúdos das questões e das escalas de respostas adotadas.

0 questionário foi validado por meio do Alpha de Cronbach, que mede a correlação global entre as variáveis utilizadas no questionário. 0 Alpha de Cronback encontrado para os vários grupos de variável foi superior a 0,70 , o que é considerado adequado. Os respectivos coeficientes (Alpha de Cronback) para cada constructo é demonstrado abaixo:

- Práticas de operacionalização $(0,886)$;

- Incentivos às atividades de MC $(0,743)$;

- Ferramentas utilizadas na análise e solução de problemas $(0,875)$; e

- Resultados das atividades de MC $(0,851)$.

Outro critério considerado relevante em uma análise multivariada é a relação de 3 para 1 entre o número de casos com o número de variáveis investigadas (GARCIA, 1995). Essa condição também foi atendida na pesquisa.

\subsection{Análises}

Os dados foram analisados por técnicas estatísticas descritivas, teste de correlação e teste de significância. A análise estatística foi realizada com o auxílio do software Statistica ${ }^{\circledR}$.

Para medir a correlação de cada variável individual (fator crítico) com as variáveis individuais do constructo resultado das atividades de melhoria (indicadores de resultado) foi utilizado o método não paramétrico de correlação de Spearman. Tal teste é recomendado quando se trabalha com variáveis qualitativas ordinais (BASILEVSKY, 1994; MONTGOMERY; RUNGER, 2003), para indicar o grau de dependência entre duas variáveis. Os coeficientes acima de 0.50 apontam correlação positiva forte, os coeficientes entre 0.30 a 0.49 indicam correlação positiva moderada. Abaixo desses valores não há evidências estatísticas de correlação ou de não haver dependência entre as variáveis.

Também foram calculados coeficientes de significância (p-valor). As correlações foram consideradas significativas quando os $p$-valores são inferiores a 0,05. Consequentemente, para valores acima de 0,05 não foi possível afirmar a existência de associação entre as variáveis.

Outra análise aplicada foi a fatorial. 0 propósito da aplicação dessa técnica foi reduzir o número de dimensões (variáveis) analisadas por meio de clusterização. Segundo Basilevsky (1994), a técnica de análise fatorial recomendada para a extração dos fatores de dados ordinais (que é o caso desta pesquisa) é a maximum likelihood. A análise fatorial e foi aplicada nos constructos do modelo conceitual mostrado na Figura 1.

A aplicação da análise fatorial possibilita responder a dois fundamentais questionamentos pertinentes à análise dessas variáveis, a saber: como fazer a redução do número de variáveis (dimensões) pesquisadas? Como explicar o comportamento de um grupo de variáveis a partir de variáveis não observadas ou latentes?

Cabe distinguir uma distribuição univariada de uma multivariada. A análise multivariada, aplicada neste trabalho, deve ser utilizada quando houver dependência entre variáveis. Essa dependência é determinada através do cálculo da covariância, que pode ser mensurado de modo adimensional por meio do coeficiente de correlação $p=0$ (BASILEVSKY, 1994). Do ponto de vista matemático, a análise multivariada diferencia-se das técnicas univariadas pela ortogonalidade entre as dimensões representadas no espaço vetorial. A análise fatorial permite, rotacionando $p$ dimensões no espaço, obter independência entre as variáveis; ou seja, construir 
da melhor maneira possível vetores ortogonais, de modo a reproduzir a estrutura original de covariância/variância.

A análise fatorial é aplicada tanto para variáveis contínuas quanto para variáveis qualitativas, ordinais e nominais. 0 uso adequado de técnicas estatísticas requer a separação das variáveis aleatórias em contínuas e discretas. Basilevsky (1994) afirma que a escolha da escala de medição depende do fenômeno ou objeto a ser estudado, do escopo e propósito da análise e da qualidade dos dados disponíveis. As escalas ordinais e nominais são classificadas como variáveis qualitativas, sendo que, em algumas situações, usa-se a suposição de continuidade das escalas ordinais.

\section{Resultados}

Neste tópico, são apresentados os resultados obtidos pela análise dos dados colhidos nas empresas. Por meio de análise fatorial e dos coeficientes de correlação de Spearman, foi possível identificar os fatores críticos para implementação de MC.

\subsection{Análise descritiva}

A Tabela 1 apresenta as práticas de operacionalização das atividades de MC mais utilizadas pelas empresas investigadas.

A pesquisa investigou como as empresas recompensam os resultados das atividades de MC. Os resultados são apresentados na Tabela 2. Apenas 37\% das empresas costumam bonificar. Geralmente, as recompensas para todo o grupo de MC e premiação de sugestões são mais utilizadas pelas empresas. Via de regra, o mecanismo de incentivo menos utilizado pelas empresas é gratificar indiretamente as melhorias alcançadas por meio de salários pagos a cada pessoa, uma vez que cerca de $60 \%$ das empresas responderam utilizar esse mecanismo com pouca ou rara frequência. Do mesmo modo, é pouco frequente o uso de recompensas diretas às pessoas através de bônus ou prêmios.

A Tabela 3 mostra as técnicas e ferramentas mais utilizadas nas atividades de MC.

Tabela 1. Meios utilizados para apoiar as atividades de melhoria contínua.

\begin{tabular}{|c|c|c|c|c|c|}
\hline & $\begin{array}{c}\text { Muito } \\
\text { frequente } \\
(\%)\end{array}$ & $\begin{array}{c}\text { Frequente } \\
(\%)\end{array}$ & $\begin{array}{c}\text { Frequência } \\
\text { mediana } \\
(\%)\end{array}$ & $\begin{array}{c}\text { Pouco } \\
\text { fequente } \\
(\%)\end{array}$ & $\begin{array}{c}\text { Raramente } \\
(\%)\end{array}$ \\
\hline Uso da ISO 9000 ou outros padrões de qualidade & 74 & 24 & 2 & 0 & 0 \\
\hline Visitas regulares ao chão de fábrica pela gerência & 50 & 26 & 11 & 13 & 0 \\
\hline Monitoramento das atividades de MC & 46 & 35 & 17 & 2 & 0 \\
\hline Emprego de política formal & 39 & 30 & 20 & 9 & 2 \\
\hline Formato genérico de solução de problemas & 35 & 39 & 11 & 11 & 4 \\
\hline Trabalho em grupo & 33 & 28 & 30 & 9 & 0 \\
\hline Comunicação face a face & 33 & 30 & 24 & 9 & 4 \\
\hline Uso de slogan & 28 & 15 & 15 & 13 & 28 \\
\hline Suporte de líder & 26 & 28 & 33 & 13 & 0 \\
\hline Plano de sugestões & 26 & 22 & 24 & 24 & 4 \\
\hline Treinamento de pessoal & 24 & 35 & 24 & 11 & 7 \\
\hline Promoção no quadro de avisos & 24 & 24 & 24 & 20 & 9 \\
\hline Suporte do staff gerencial & 20 & 33 & 35 & 13 & 0 \\
\hline Promoção por mídia interna & 15 & 28 & 22 & 22 & 13 \\
\hline Sistemas de incentivo & 13 & 17 & 24 & 24 & 22 \\
\hline Uso de Manutenção Preventiva Total & 11 & 22 & 35 & 15 & 17 \\
\hline Prêmios da qualidade & 11 & 7 & 24 & 35 & 24 \\
\hline Promoção por competição e prêmio & 7 & 17 & 20 & 24 & 33 \\
\hline
\end{tabular}

Tabela 2. Mecanismos adotados de incentivos às atividades de melhoria contínua.

\begin{tabular}{lccccc}
\hline & $\begin{array}{c}\text { Muito } \\
\text { frequente } \\
(\%)\end{array}$ & $\begin{array}{c}\text { Frequente } \\
(\%)\end{array}$ & $\begin{array}{c}\text { Frequência } \\
\text { mediana } \\
(\%)\end{array}$ & $\begin{array}{c}\text { Pouco } \\
\text { frequente } \\
(\%)\end{array}$ & $\begin{array}{c}\text { Raramente } \\
(\%)\end{array}$ \\
\hline Recompensas indiretas por meio de salários individuais & 9 & 11 & 22 & 24 & 35 \\
Recompensas diretas por meio de bônus/prêmios & 9 & 22 & 13 & 30 & 26 \\
Sugestões são avaliadas e recompensadas & 15 & 26 & 11 & 33 & 15 \\
Resultados das melhorias são recompensados à todo o time & 13 & 28 & 17 & 26 & 15 \\
Sistemas de incentivo & 11 & 26 & 22 & 30 & 11 \\
\hline
\end{tabular}


Tabela 3. Análise do uso de técnicas de solução de problemas utilizadas nas atividades de melhoria contínua.

\begin{tabular}{|c|c|c|c|c|c|}
\hline & $\begin{array}{c}\text { Muito } \\
\text { frequente } \\
(0)\end{array}$ & $\begin{array}{c}\text { Frequente } \\
(\%)\end{array}$ & $\begin{array}{l}\text { Frequência } \\
\text { média } \\
(\%)\end{array}$ & $\begin{array}{c}\text { Pouco } \\
\text { frequente } \\
(\%)\end{array}$ & $\begin{array}{c}\text { Raramente } \\
(\%)\end{array}$ \\
\hline Ferramentas de padronização & 54 & 30 & 13 & 0 & 2 \\
\hline Ferramentas de identificação de problemas & 33 & 28 & 26 & 7 & 7 \\
\hline Programa 5S & 30 & 39 & 20 & 11 & 0 \\
\hline Sete ferramentas básicas da qualidade & 24 & 43 & 15 & 11 & 7 \\
\hline Ferramentas de mapeamento de processos & 24 & 33 & 24 & 9 & 11 \\
\hline CEP (Controle Estatístico de Processo) & 22 & 15 & 33 & 13 & 17 \\
\hline FMEA (Análise do Modo e Efeito de Falhas) & 15 & 15 & 24 & 26 & 20 \\
\hline Sete ferramentas gerenciais da qualidade & 11 & 13 & 13 & 35 & 28 \\
\hline Simulação & 11 & 17 & 30 & 17 & 24 \\
\hline Ferramentas de visualização & 9 & 24 & 17 & 28 & 22 \\
\hline Ferramentas de criatividade & 7 & 35 & 15 & 28 & 15 \\
\hline Seis Sigma & 7 & 9 & 22 & 11 & 52 \\
\hline QFD & 4 & 7 & 26 & 22 & 41 \\
\hline
\end{tabular}

A técnica mais utilizada são as ferramentas de padronização. Nessa categoria são incluídos os procedimentos, rotinas e planos de controle da qualidade. Na sequência, são apontadas as ferramentas de identificação de problemas e o Programa 5S. As técnicas menos utilizadas são o QFD e a abordagem Seis Sigma, o que pode ser explicado pela maior complexidade dessas duas ferramentas e, portanto, da necessidade de conhecimentos mais especializados em suas aplicações.

Quando questionados sobre o nível de dificuldade que as empresas enfrentam na condução de suas atividades de MC, foram destacadas três situações (assinaladas como muito difícil ou difícil na Tabela 4). Para 48\% das empresas, a maior dificuldade é difundir os esforços de melhoria de forma a aumentar o grau de participação dos colaboradores nas atividades de MC. Outra grande dificuldade é dar início ao programa de melhoria, situação apontada por 45\% dos entrevistados. A terceira dificuldade é gerenciar vários projetos simultaneamente (44\%). Em contraponto, a atividade considerada como mais fácil é levantar recursos suficientes para a execução dos projetos.

\subsection{Fatores críticos das atividades de $M C$}

Análises de correlações foram realizadas para verificar o relacionamento entre as variáveis individuais de cada constructo (práticas de operacionalização, incentivos às atividades de MC e ferramentas utilizadas na análise e solução de problemas) com indicadores de resultado das atividades de MC.

$\mathrm{Na}$ Tabela 5, das 18 variáveis relacionadas à práticas de operacionalização, apenas quatro apresentaram coeficientes de correlação estatis- ticamente significativos (acima de 0,30 e p-valor menor que 0,05). Corroborando o trabalho de Harrison (2000) verificou-se que a prática de treinamento de pessoal em ferramentas de solução de problemas tem impacto positivo no resultado nas atividades de MC, por contribuírem para o aumento da produtividade e para o desenvolvimento das habilidades dos empregados.

A mesma situação foi observada para a existência de planos de sugestão na empresa com contribuições para melhoria desses dois indicadores de desempenho. 0 objetivo dos planos de sugestão é desenvolver, nos empregados, a autodisciplina e sua participação na busca por melhorias no sistema produtivo, sobretudo em termos de qualidade.

A comunicação face a face apresentou correlação positiva com os indicadores de resultado relacionados à satisfação dos clientes e ao desenvolvimento das habilidades dos empregados. Nota-se convergência com afirmações de diversos autores sobre fatores comportamentais pesquisados na literatura (BESSANT; FRANCIS, 1999; SAVOLAINEN, 1999; MURRAY; CHAPMAN, 2003; DABHILKAR; BENGTSSON, 2004; ABRAHAMSSON; GERDIN, 2006). A utilização dessa forma de comunicação estimularia a interação entre os empregados, facilitaria a troca de informações e contribuiria para a resolução de problemas nas empresas. De acordo com Terziovski e Sohal (2000) outra prática de operacionalização neste constructo foram as visitas regulares ao chão de fábrica feitas pelos gerentes, com influência no indicador de resultado das habilidades dos funcionários. As visitas normalmente têm o objetivo de acompanhar as rotinas de trabalho, identificar pontos de melhoria e repassar instruções. 
Tabela 4. Principais dificuldades nas atividades de MC.

\begin{tabular}{lccccc}
\hline & $\begin{array}{c}\text { Muito difícil } \\
(\%)\end{array}$ & $\begin{array}{c}\text { Difícil } \\
(\%)\end{array}$ & $\begin{array}{c}\text { Dificuldade mediana } \\
(\%)\end{array}$ & $\begin{array}{c}\text { Fácil } \\
(\%)\end{array}$ & $\begin{array}{c}\text { Muito fácil } \\
(\%)\end{array}$ \\
\hline Iniciar mudanças & 2 & 43 & 26 & 28 & 0 \\
Manter esforços de atividades em andamento & 4 & 30 & 43 & 20 & 2 \\
Difundir esforços de mudanças & 2 & 46 & 26 & 24 & 2 \\
Gerenciar vários projetos & 7 & 37 & 41 & 15 & 0 \\
Alinhar atividades de MC e estratégias & 2 & 20 & 46 & 26 & 7 \\
Levantar recursos suficientes & 4 & 20 & 33 & 39 & 4 \\
\hline
\end{tabular}

Tabela 5. Coeficientes de correlação entre os meios de apoio às atividades de MC e resultados.

\begin{tabular}{|c|c|c|c|c|c|c|}
\hline & Produtividade & Qualidade & Lead time & Custo & $\begin{array}{l}\text { Satisfação } \\
\text { do cliente }\end{array}$ & $\begin{array}{c}\text { Habilidade } \\
\text { dos } \\
\text { empregados }\end{array}$ \\
\hline Uso de slogan & 0,19 & $-0,05$ & 0,28 & 0,10 & 0,25 & 0,33 \\
\hline $\begin{array}{l}\text { Treinamento de pessoal em ferramentas de } \\
\text { solução de problemas }\end{array}$ & $0,40^{*}$ & 0,14 & 0,37 & 0,35 & 0,23 & $0,50^{*}$ \\
\hline Monitoramento das atividades de MC & 0,10 & 0,13 & 0,02 & 0,10 & 0,17 & 0,26 \\
\hline Suporte do staff gerencial & 0,18 & 0,07 & 0,32 & 0,32 & 0,13 & 0,36 \\
\hline Sistemas de incentivo & 0,21 & $-0,15$ & 0,05 & 0,17 & $-0,16$ & 0,17 \\
\hline Liderança que dá suporte & 0,01 & 0,10 & 0,21 & 0,07 & 0,37 & 0,39 \\
\hline Trabalho em grupo & 0,16 & 0,18 & 0,14 & 0,13 & 0,08 & 0,34 \\
\hline Plano de sugestões & $0,42^{*}$ & 0,15 & 0,27 & 0,33 & 0,09 & $0,49^{*}$ \\
\hline Formato genérico de solução de problemas & 0,31 & 0,22 & 0,32 & 0,30 & 0,07 & 0,21 \\
\hline Promoção no quadro de avisos & 0,29 & 0,16 & 0,19 & 0,17 & 0,17 & 0,32 \\
\hline Promoção por mídia interna & 0,29 & 0,15 & 0,28 & 0,21 & 0,12 & 0,31 \\
\hline Emprego de política formal & 0,21 & $-0,09$ & 0,23 & 0,00 & 0,00 & 0,23 \\
\hline Comunicação face a face & 0,23 & 0,22 & 0,27 & 0,21 & 0,57 & $0,49^{*}$ \\
\hline Visitas regulares ao chão de fábrica & 0,10 & 0,15 & 0,36 & 0,05 & 0,23 & $0,43^{*}$ \\
\hline $\begin{array}{l}\text { Uso do ISO 9000:2000 ou outros padrões de } \\
\text { qualidade }\end{array}$ & 0,09 & 0,14 & 0,19 & 0,16 & 0,20 & 0,25 \\
\hline Uso de Manutenção Preventiva Total & 0,25 & 0,10 & 0,21 & 0,09 & 0,15 & 0,37 \\
\hline Prêmios da qualidade & 0,18 & $-0,07$ & 0,21 & 0,12 & 0,17 & 0,21 \\
\hline Promoção por competição e prêmio & 0,31 & 0,21 & 0,11 & 0,22 & 0,26 & 0,28 \\
\hline
\end{tabular}

*Nivel de significância $\mathrm{p} \leq 0,05$.

Quanto à análise dos incentivos (Tabela 6), de forma geral, é constatada que a existência de um sistema de incentivos tem influência positiva no aumento da produtividade, na redução do lead time e no desenvolvimento das habilidades dos empregados. Os coeficientes de correlação para práticas mais específicas de recompensas não indicaram associação com qualquer indicador de resultado de MC, contrariando a importância dada a esse item por Atkinson (1994).

$\mathrm{Na}$ Tabela 6, conclui-se que as empresas deveriam se empenhar na formalização de sistemas de incentivos, pois esta prática demonstra estar associada com o desempenho das atividades de MC.

$\mathrm{Na}$ Tabela 7, oito ferramentas apresentam evidências estatísticas de contribuição para melhorar o desempenho das atividades de MC: as ferramentas de identificação de problemas, as sete ferramentas básicas da qualidade, as sete ferramentas gerenciais da qualidade, as ferramentas de mapeamento de processos, QFD, as ferramentas de visualização, técnicas de simulação e o Controle Estatístico de Processo.

As ferramentas de mapeamento de processos apresentaram correlação positiva com o indicador qualidade e as técnicas de simulação contribuem para o desenvolvimento das habilidades dos empregados. Em concordância com Terziovsky e Sohal (2000) QFD, CEP e técnicas de simulação não são empregados com frequência pelas empresas da amostra (Tabela 3), porém as três foram relacionadas como importantes para o desempenho das atividades de MC. Também de acordo com esses autores, FMEA, técnicas de criatividade, ferramentas de padronização, Programa $5 \mathrm{~S}$ e Seis Sigma não apresentaram evidências estatísticas de sua contribuição para os resultados das atividades de MC das empresas investigadas. 
Tabela 6. Coeficientes de correlação entre incentivos e resultados.

\begin{tabular}{|c|c|c|c|c|c|c|}
\hline & Produtividade & Qualidade & Lead time & Custo & $\begin{array}{l}\text { Satisfação } \\
\text { do cliente }\end{array}$ & $\begin{array}{l}\text { Habilidade dos } \\
\text { empregados }\end{array}$ \\
\hline Sugestões são avaliadas e recompensadas & 0,29 & 0,01 & 0,19 & 0,08 & 0,02 & 0,28 \\
\hline Recompensas diretas por meio de bônus/prêmios & 0,18 & $-0,07$ & 0,18 & $-0,07$ & $-0,07$ & 0,12 \\
\hline Recompensas diretas por meio de salários individuais & 0,20 & 0,12 & 0,09 & $-0,07$ & $-0,16$ & 0,16 \\
\hline Sistemas de incentivo & $0,30^{*}$ & $-0,01$ & $0,40^{*}$ & 0,14 & 0,25 & $0,37^{*}$ \\
\hline $\begin{array}{l}\text { Resultados das melhorias são recompensados } \\
\text { à todo o time }\end{array}$ & 0,09 & $-0,13$ & 0,01 & $-0,10$ & $-0,12$ & $-0,06$ \\
\hline
\end{tabular}

*Nível de significância $\mathrm{p} \leq 0,05$.

Tabela 7. Coeficientes de correlação entre ferramentas e resultados.

\begin{tabular}{lcccccc} 
& Produtividade & Qualidade & Lead time & $\begin{array}{c}\text { Custo } \\
\text { Satisfação do } \\
\text { cliente }\end{array}$ & $\begin{array}{c}\text { Habilidade dos } \\
\text { empregados }\end{array}$ \\
\hline Ferramentas de identificação de problemas & $0,29^{*}$ & $0,32^{*}$ & $0,47^{*}$ & 0,23 & $0,29^{*}$ & $0,32^{*}$ \\
Sete ferramentas básicas da qualidade & 0,29 & 0,13 & 0,11 & 0,18 & $0,32^{*}$ & 0,28 \\
Sete ferramentas gerenciais da qualidade & 0,1 & 0,22 & 0,11 & $-0,03$ & $0,30^{*}$ & 0,29 \\
Ferramentas de mapeamento de processos & 0,18 & $0,37^{*}$ & 0,12 & 0,04 & 0,20 & 0,27 \\
FMEA & 0,12 & $-0,06$ & $-0,03$ & $-0,03$ & 0,24 & 0,00 \\
QFD & 0,12 & 0,25 & 0,27 & 0,03 & $0,37^{*}$ & 0,23 \\
Ferramentas de criatividade & 0,22 & 0,16 & 0,23 & 0,17 & 0,25 & 0,22 \\
Ferramentas de visualização & 0,11 & 0,2 & 0,15 & 0,07 & $0,33^{*}$ & 0,29 \\
Ferramentas de padronização & 0,25 & 0,18 & 0,19 & 0,17 & 0,16 & 0,31 \\
Programa 5S & 0,2 & $-0,05$ & 0,25 & 0,04 & 0,08 & 0,15 \\
Simulação & 0,08 & 0,25 & 0,26 & 0,05 & 0,18 & $0,34^{*}$ \\
Seis Sigma & 0,14 & $-0,02$ & 0,20 & 0,05 & 0,07 & 0,23 \\
CEP & 0,04 & 0,11 & 0,14 & 0,07 & $0,31^{*}$ & $0,07^{*}$ \\
\hline
\end{tabular}

*Nível de significância $p \leq 0,05$.

\subsection{Análise fatorial}

A Tabela 8 mostra a extração de dois fatores referentes às variáveis relacionadas ao constructo "práticas de operacionalização" das atividades de MC. A partir da análise, foram obtidos dois grupos de variáveis do constructo: a promoção da $M C$ (fator 1) e o suporte e liderança (fator 2).

Com o objetivo de identificar elementos centrais das práticas de operacionalização das atividades de MC, deve-se analisar as estruturas dos fatores extraídos pela análise fatorial. A começar pelo primeiro fator (fator 1), estão associadas, pela ordem de relevância, as seguintes dimensões: 1) emprego de política formal de incentivos; 2) plano de sugestões; 3) uso de slogan; 4) promoção por mídia interna; e 5) a utilização do modelo do prêmio nacional da qualidade. Contrapondo-se ao fator 1 , tem-se no fator 2 as seguintes dimensões pela relevância: 1) liderança; 2) visitas regulares ao chão de fábrica; 3) suporte do staff gerencial; e 4) comunicação face a face.

Deste modo é possível fazer a seguinte classificação das dimensões que compõem o constructo: a) associado ao fator 1, há a promoção da MC; e b) ao fator 2, o suporte e liderança às práticas das atividades de MC. A principal dimensão relacionada à promoção das práticas de $\mathrm{MC}$ é a formalização de políticas de incentivos. Os incentivos não são necessariamente financeiros, podem ser um reconhecimento formal através de promoções ou distinção perante a organização. Algumas empresas associam o plano de carreira à participação em projetos de inovação e melhoria. Quanto ao suporte e liderança do processo de melhoria, destacam-se a liderança gerencial e as visitas regulares ao chão de fábrica pela gerência. Essas dimensões mostram a importância da gerência na condução dos programas e processos de melhoria.

Portanto, as variáveis do constructo de práticas de operacionalização das atividades de MC podem ser reduzidas a duas dimensões: i) promoção; e ii) liderança. Tanto para uma quanto para outra elas podem ser operacionalizadas pelas variáveis associadas à análise fatorial, que estão destacadas na Tabela 8.

Da análise fatorial aplicada às ferramentas de MC, mostrada na Tabela 9, dois fatores foram extraídos. A classificação obtida distingue ferramentas de padronização, no fator 1 , das ferramentas avançadas de qualidade e comunicação, fator 2. As ferramentas de padronização são aplicadas na fase final do ciclo PDCA (Plan, Do, Check, Action) e as do fator 2 nas fases iniciais. 
Tabela 8. Análise fatorial das práticas de operacionalização das atividades de MC.

\begin{tabular}{|c|c|c|}
\hline Operacionalização & $\begin{array}{l}\text { Fator 1: } \\
\text { promoção } \\
\text { da MC }\end{array}$ & $\begin{array}{l}\text { Fator 2: suporte } \\
\text { e liderança do } \\
\text { processo de MC }\end{array}$ \\
\hline Uso de slogan & 0,650726 & 0,250196 \\
\hline $\begin{array}{l}\text { Treinamento de pessoal em } \\
\text { ferramentas de solução de } \\
\text { problemas }\end{array}$ & 0,559092 & 0,560123 \\
\hline $\begin{array}{l}\text { Monitoramento das } \\
\text { atividades de MC }\end{array}$ & 0,231542 & 0,583534 \\
\hline Suporte do staff gerencial & 0,129103 & 0,649661 \\
\hline Sistemas de incentivo & 0,546407 & 0,088659 \\
\hline Liderança que dá suporte & 0,127316 & 0,801790 \\
\hline Trabalho em grupo & 0,557305 & 0,447761 \\
\hline Plano de sugestões & 0,683911 & 0,183614 \\
\hline $\begin{array}{l}\text { Formato genérico de } \\
\text { solução de problemas }\end{array}$ & 0,254060 & 0,450827 \\
\hline $\begin{array}{l}\text { Promoção no quadro } \\
\text { de avisos }\end{array}$ & 0,519019 & 0,282805 \\
\hline Promoção por mídia interna & 0,646867 & 0,235368 \\
\hline $\begin{array}{l}\text { Emprego de política } \\
\text { formal de incentivos }\end{array}$ & 0,891343 & $-0,085310$ \\
\hline Comunicação face a face & 0,112776 & 0,617558 \\
\hline $\begin{array}{l}\text { Visitas regulares ao } \\
\text { chão de fábrica }\end{array}$ & 0,128029 & 0,665769 \\
\hline $\begin{array}{l}\text { Uso do 1S0 9000:2000 } \\
\text { ou outros padrões de } \\
\text { qualidade }\end{array}$ & 0,062867 & 0,539872 \\
\hline $\begin{array}{l}\text { Uso de Manutenção } \\
\text { Preventiva Total; }\end{array}$ & 0,476350 & 0,233464 \\
\hline Prêmios da qualidade & 0,615319 & 0,088866 \\
\hline $\begin{array}{l}\text { Promoção por competição } \\
\text { e prêmio }\end{array}$ & 0,210354 & 0,264041 \\
\hline
\end{tabular}

Tabela 9. Análise fatorial das ferramentas das atividades de $\mathrm{MC}$.

\begin{tabular}{|c|c|c|}
\hline Ferramentas & $\begin{array}{c}\text { Fator 1: } \\
\text { padronização }\end{array}$ & $\begin{array}{l}\text { Fator 2: ferramentas } \\
\text { avançadas de qualidade } \\
\text { e comunicação }\end{array}$ \\
\hline $\begin{array}{l}\text { Ferramentas de } \\
\text { identificação de } \\
\text { problemas }\end{array}$ & 0,375315 & 0,427750 \\
\hline $\begin{array}{l}\text { Sete ferramentas } \\
\text { básicas da qualidade }\end{array}$ & 0,466712 & 0,489165 \\
\hline $\begin{array}{l}\text { Sete ferramentas } \\
\text { gerenciais da } \\
\text { qualidade }\end{array}$ & 0,280851 & 0,604485 \\
\hline $\begin{array}{l}\text { Ferramentas de } \\
\text { mapeamento de } \\
\text { processos }\end{array}$ & 0,550255 & 0,351815 \\
\hline FMEA & $-0,068393$ & 0,710326 \\
\hline QFD & $-0,110124$ & 0,670373 \\
\hline $\begin{array}{l}\text { Ferramentas de } \\
\text { criatividade }\end{array}$ & 0,369338 & 0,550016 \\
\hline $\begin{array}{l}\text { Ferramentas de } \\
\text { visualização }\end{array}$ & 0,239657 & 0,676942 \\
\hline $\begin{array}{l}\text { Ferramentas de } \\
\text { padronização }\end{array}$ & 0,955007 & $-0,049943$ \\
\hline Programa 5S & 0,163211 & 0,365050 \\
\hline Simulação & 0,078548 & 0,649792 \\
\hline Seis Sigma & 0,158598 & 0,625077 \\
\hline CEP & 0,077022 & 0,778774 \\
\hline
\end{tabular}

Esse é um modo de classificação baseado da extração de fatores, que indica quais ferramentas tiveram certas similaridades de respostas. É coerente separar nas duas categorias, visto que ferramentas de padronização têm a finalidade da reprodutibilidade dos métodos e procedimentos de controle após a identificação e proposta de solução dos problemas.

A Tabela 10 mostra a possibilidade de redução do número de dimensões relacionadas a resultados decorrentes da MC. Obteve-se a relação lógica entre custos de produção e produtividade, correspondente ao fator 1. Outra menos previsível, obtida no fator 2, relaciona habilidades e satisfação dos clientes. Portanto, foi possível, pela análise fatorial, separar os resultados em duas categorias: a dos financeiros e a dos não financeiros.

Somente um fator foi gerado na análise das dimensões relacionadas aos sistemas de incentivos, cujos resultados da análise são mostrados na Tabela 11. Duas dimensões (variáveis) poderiam ser reduzidas em uma única, são elas: 1) sugestões são avaliadas e recompensadas; e 2) resultados das melhorias são recompensados diretamente através de bônus/prêmios.

Como indica a Tabela 12, as práticas da operacionalização, com impacto estatisticamente significativo nos resultados financeiros, são: treinamento do pessoal em ferramentas de solução de problemas e adoção de planos de sugestão. 0 treinamento em ferramentas de solução de problemas tem impacto tanto nos resultados financeiros quanto nos não financeiros. Suporte e liderança do processo de melhoria têm maior efeito nos resultados não financeiros.

Tabela 10. Análise fatorial das medidas de resultados obtidos com as atividades de MC.

\begin{tabular}{lcc}
\hline \multicolumn{1}{c}{ Resultados } & $\begin{array}{c}\text { Fator 1: } \\
\text { financeiro }\end{array}$ & $\begin{array}{c}\text { Fator 2: não } \\
\text { financeiro }\end{array}$ \\
\hline Produtividade & 0,984032 & 0,109513 \\
Qualidade & 0,201742 & 0,522038 \\
Lead time & 0,330915 & 0,511209 \\
Custo & 0,716037 & 0,210722 \\
Satisfação do cliente & 0,173483 & 0,617040 \\
Habilidade dos empregados & 0,242785 & 0,716520 \\
\hline
\end{tabular}

Tabela 11. Análise fatorial das dimensões associadas aos sistemas de incentivo às atividades de MC.

\begin{tabular}{lc}
\hline \multicolumn{1}{c}{ Sistemas de Incentivo } & Fator \\
\hline Sugestões são avaliadas e recompensadas & $-0,750205$ \\
Resultados das melhorias são recompensados & $-0,909481$ \\
diretamente através de bônus/prêmios & \\
$\begin{array}{l}\text { Resultados das melhorias são recompensados } \\
\text { indiretamente através de salários individuais }\end{array}$ & $-0,504846$ \\
$\begin{array}{l}\text { Resultados das melhorias são recompensados } \\
\text { a toda a equipe }\end{array}$ & $-0,490578$ \\
Sistemas de incentivo & $-0,343735$ \\
\hline
\end{tabular}


Correlacionando os fatores obtidos pela análise fatorial com o impacto nos resultados, a percepção dos respondentes indica que as práticas, sistemas de incentivos e as ferramentas de solução de problemas têm impactos positivos não financeiros; por exemplo, na habilidade dos funcionários e na satisfação dos clientes. Os resultados dessa correlação estão na Tabela 13, que mostra também que uso de práticas de promoção da MC também tem efeitos nos resultados financeiros.

Os níveis de significância calculados indicam que a adoção de práticas de suporte e liderança para a MC e o uso de ferramentas de suporte para a solução de problemas têm maior efeito nos resultados não financeiros (nas habilidades dos funcionários e na satisfação dos clientes).

Tabela 12. Análise de correlação de Spearman entre as práticas de MC e resultados.

\begin{tabular}{|c|c|c|}
\hline Práticas de Operacionalização & Financeiro & Não financeiro \\
\hline Uso de slogan & 0,141503 & 0,299678 \\
\hline $\begin{array}{l}\text { Treinamento de pessoal em } \\
\text { ferramentas de solução de problemas }\end{array}$ & $0,407950^{*}$ & $0,478026^{*}$ \\
\hline Monitoramento das atividades de $\mathrm{MC}$ & 0,122943 & 0,175855 \\
\hline Suporte do staff gerencial & 0,246613 & 0,335577 \\
\hline Sistemas de incentivo & 0,208061 & 0,033271 \\
\hline Liderança que dá suporte & 0,064529 & $0,421456^{*}$ \\
\hline Trabalho em grupo & 0,148111 & 0,187807 \\
\hline Plano de sugestões & $0,408010^{*}$ & 0,294237 \\
\hline $\begin{array}{l}\text { Formato genérico de solução de } \\
\text { problemas }\end{array}$ & 0,238367 & 0,220973 \\
\hline Promoção no quadro de avisos & 0,238785 & 0,265348 \\
\hline Promoção por mídia interna & 0,258433 & 0,253482 \\
\hline $\begin{array}{l}\text { Emprego de política formal de } \\
\text { incentivos }\end{array}$ & 0,130892 & 0,143175 \\
\hline Comunicação face a face & 0,270488 & $0,567219^{*}$ \\
\hline Visitas regulares ao chão de fábrica & 0,030170 & $0,489207^{*}$ \\
\hline $\begin{array}{l}\text { Uso do 1S0 9000:2000 ou outros } \\
\text { padrões de qualidade }\end{array}$ & 0,131876 & 0,250140 \\
\hline Uso de Manutenção Preventiva Total; & 0,216618 & 0,294286 \\
\hline Prêmios da qualidade & 0,157136 & 0,173723 \\
\hline Promoção por competição e prêmio & 0,239376 & 0,322479 \\
\hline
\end{tabular}

Tabela 13. Análise de correlação de Spearman entre os fatores identificados na análise fatorial e a percepção no impacto nos resultados financeiros e não financeiros.

\begin{tabular}{|c|c|c|}
\hline Fatores críticos & $\begin{array}{l}\text { Resultados } \\
\text { financeiros }\end{array}$ & $\begin{array}{c}\text { Resultados } \\
\text { não financeiros }\end{array}$ \\
\hline Uso ferramentas de padronização & 0,095265 & $0,318785^{* * *}$ \\
\hline $\begin{array}{l}\text { Uso ferramentas de suporte a } \\
\text { solução de problemas }\end{array}$ & 0,051258 & $0,461919^{* * *}$ \\
\hline $\begin{array}{l}\text { Uso de mecanismos de } \\
\text { recompensa direta }\end{array}$ & 0,156828 & 0,126592 \\
\hline Uso de práticas de promoção da $\mathrm{MC}$ & $0,288360^{*}$ & $0,322118^{* * *}$ \\
\hline $\begin{array}{l}\text { Uso de práticas de suporte e } \\
\text { liderança para a MC }\end{array}$ & 0,205457 & $0,583094^{* * * *}$ \\
\hline
\end{tabular}

****ível de significância $p \leq 0,1 ;{ }^{* *}$ Nível de significância $0,1<p \leq 0,4$; e *Nivel de significância $0,4<p \leq 0,6$.

\section{Conclusões}

0 modelo conceitual elaborado para a pesquisa sugere relações de dependência entre as variáveis individuais alocadas em três constructos (práticas de operacionalização, sistemas de incentivos e ferramentas de apoio às atividades de $\mathrm{MC}$ ) e indicadores de resultado da MC (produtividade, qualidade, redução de lead time, custo, satisfação do cliente e desenvolvimento de habilidades dos empregados). As variáveis estatisticamente significativas podem ser consideradas como fatores críticos, pois exercem impactos positivos nos resultados das atividades de MC.

Foram identificados fatores críticos como: o treinamento em ferramentas de solução de problemas, o incentivo a sugestões, a utilização de comunicação face a face, o uso de visitas ao chão de fábrica e adoção de sistemas de incentivos. Esses resultados vão ao encontro de muitos dos fatores críticos apontados em publicações sobre melhoria contínua (YOUSSEF; ZAIRI, 1995; TERZIOVSKY; SOHAL, 2000; JABNOUN, 2001; LEE, 2004; FRYER; ANTONY; DOUGLAS, 2007).

Algumas práticas de operacionalização influenciam positivamente os resultados das atividades de MC. Entre elas a comunicação aberta, a participação dos empregados nas atividades de melhoria e o uso de mecanismos de incentivos. Essas práticas certamente contribuem para a formação de uma cultura voltada para maior engajamento da empresa com as atividades de MC.

Outro resultado obtido na pesquisa é que o desenvolvimento dos recursos humanos apresenta-se como um fator de vital importância para o sucesso de qualquer programa de MC. Muitas das práticas listadas na Tabela 1 e utilizadas com frequência pelas empresas (comunicação face a face, trabalho em equipe, treinamento pessoal, suporte gerencial, estímulo a sugestões) apontam para a formação de comportamentos que favorecem as atividades de MC.

Pode-se constatar que as atividades de MC estão concentradas nas áreas produtivas da empresa (qualidade, produção, engenharia), o que indica a prevalência de atividades de melhoria locais visando a resolução de problemas e não necessariamente uma abordagem mais ampla voltada para a estratégia da empresa.

As empresas devem estar atentas para que os programas de capacitação (utilizados com frequência por 59\% delas) não sejam voltados apenas para o treinamento de técnicas e ferramentas de melhoria. Os programas devem abordar questões associadas ao desenvolvimento de habilidades 
pessoais fundamentais para a MC. Verificou-se que treinamento do pessoal na solução de problemas tem relação estatisticamente significativa com o aumento da produtividade e com o desenvolvimento das habilidades dos empregados. Por isso, as empresas devem investir na capacitação em ferramentas aplicadas à MC, uma vez que sua utilização afeta positivamente os resultados das atividades de MC, além de dotar os empregados com habilidades apropriadas.

Em outros termos, o treinamento em ferramentas de solução de problemas afeta diversos resultados financeiros, como custo de produção e produtividade do pessoal. Tal treinamento também influencia resultados não financeiros, que estão associados à satisfação dos clientes e habilidades dos funcionários (esses resultados são mostrados na Tabela 11).

As análises estatísticas, em sua maioria, indicam coeficientes de correlação moderados para as ferramentas de identificação de problemas nos indicadores de resultado observados. A MC não deve ser desconectada das técnicas; são elas que capacitam as empresas para desenvolver competências e habilidades dos colaboradores. Nesse sentido, foi possivel classificar as técnicas de apoio à solução de problemas em dois tipos, conforme indica a análise fatorial mostrada na Tabela 9: i) as de padronização, necessárias na consolidação da implantação das soluções dos problemas; e ii) as ferramentas avançadas de qualidade e comunicação, que abrangem a identificação de problemas e suas causas. Ferramentas de padronização e de identificação de problemas, o Programa $5 \mathrm{~S}$ e as sete ferramentas da qualidade são as mais difundidas. Porém, técnicas como QFD e Seis Sigmas que são mais complexas e exigem conhecimentos mais especializados são utilizadas com frequência por menos de 20\% das empresas da amostra. Portanto, os resultados indicam que as empresas fazem o uso mais frequente de ferramentas de menor grau de complexidade, o que poderia indicar que elas estão nos primeiros estágios de evolução da gestão da MC. Porém, essa observação requer acompanhamento mais profundo das práticas das empresas.

Como limitação, ainda que se observe relativa confiabilidade dos constructos, estes ainda são sujeitos a melhorias, incorporando-se novas dimensões a eles. Outra limitação decorre da abrangência do tema tratado, sujeito a grande número de variáveis subjetivas, o que implica na necessidade de serem incorporados novos temas relacionados às atividades de $\mathrm{MC}$, que não fizeram parte do escopo deste trabalho, mas que podem ser considerados em trabalhos futuros. Além destas, outra limitação consiste no fato de a amostra ter sido baseada somente em empresas que possuem ISO 9000, não possibilitando, dessa forma, generalizar, analisar o impacto de certificações de qualidade sobre as questões aqui levantadas quanto à MC. Esta lacuna pode ser explorada em trabalhos futuros sobre o tema.

Pesquisas futuras podem analisar em profundidade algumas das relações entre as variáveis dependentes e independentes aqui observadas. Novos trabalhos com outros métodos de investigação, como entrevistas em profundidade, que podem ser aplicadas para determinar uma lista definitiva de fatores críticos para condução das atividades de melhoria. Além dessas alternativas, estudos setoriais devem ser incentivados.

\section{Referências}

ABRAHAMSSON, G.; GERDIN, J. Exploiting institutional contradictions The role of management accounting in continuous t implementation. Qualitative Research in Accounting \& Management, v. 3, n. 2, p. 126-144. 2006.

ATKINSON, C. Continuous Improvement: The ingredients of change. International Journal of Contemporary Hospitality Management, v. 6, n. 2, p. 6-8, 1994.

ATTADIA, L.; MARTINS, R. A. A medição de desempenho como base para a evolução da MC: um estudo teórico. Produção, v. 13, n. 2, p. 33-41, 2003.

AUGSDORFER, P; HARDING, R. Changing competitive forces in Europe: improvement in a sample of French, German and British companies. European Business Review, v. 95, n. 4, p. 3-9, 1995.

BASILEVSKY, A. Statistical factor analysis and related methods: theory and applications. New York: Wiley Interscience, 1994.

BECHET, A. J.; WAINWRIGHT, C. E. R.; BANCE, D. Implementing an industrial continuous improvement system: a knowledge management case study. Industrial Management \& Data Systems, v. 100, n. 7, p. 330-338, 2000.

BESSANT, J. et al. Rediscovering continuous improvement. Technovation, v. 14, n. 1, p. 17-29, 1994.

BESSANT, J.; CAFFYN, S. High involvement innovation. International Journal of Technology and Management, v. 14, n. 1, 1997.

BESSANT, J.; CAFFYN, S.; GALLAGHER, M. An evolutionary model of continuous improvement behaviour. Technovation, v. 21, n. 2, p. 67-77, 2001.

BESSANT, J.; FRANCIS, D. Developing strategic continuous improvement capability, International Journal of Operations \& Production Management, v. 19, n. 11, p. 1106-1119, 1999.

BOER, H. et al. Changes from suggestion box to organisational learning: continuous improvement in Europe and Australia. Aldershot: Ashgate, 2000.

BOND, T. C. The role of performance measurement in continuous improvement. International Journal of 
Operations \& Production Management, v. 19, n. 12, p. 13-18, 1999.

CAFFYN, S. Development of a continuous improvement selfassessment tool. International Journal of Operations and Production Management, v. 19, n. 11, p. 38-53, 1999.

CHAPMAN, R. L.; HYLAND, P. W. Continuous improvement strategies across selected Australian manufacturing sectors. Benchmarking for Quality Management \& Technology, v. 4, n. 3, p. 175-188, 1997.

DABHILKAR, M.; BENGTSSON, L. Balanced scorecards for strategic and sustainable continuous improvement capability. Journal of Manufacturing Technology Management of the company, v. 15, n. 4, p. 350-359, 2004.

DELBRIDGE, R.; BARTON, H. Organizing for continuous improvement: structures and roles in automotive components plants. International Journal of Operations \& Production Management, v. 22, n. 6, p. 680-692, 2002.

FAGER, B. et al. Enabling continuous improvement: a case study of implementation. Journal of Manufacturing Technology Management, v. 15, n. 4, p. 315-324, 2004.

FRYER, K. J.; ANTONY, J.; DOUGLAS, A. Critical success factors of continuous improvement in the public sector. The TQM Magazine, v. 9, n. 5, p. 497-517, 2007.

GARCIA, J. G. Análise de la información mercadológica através de la estatística multivariante. Ciudad de Mexico: Alambra Mexicana, 1995. 235 p.

HARRINGTON, H. J. Continuous versus breakthrough improvement: finding the right answer. Business Process Re-engineering \& Management Journal, v. 1, n. 3, p. 31-49, 1995.

HARRISON, A. Continuous improvement: the trade-off between self management and discipline. Integrated Manufacturing Systems, v. 11, n. 3, p. 180-187, 2000.

HYLAND, P. W. et al. A comparison of Australian firms and their use of continuous. improvement tools. The TQM Magazine, v. 12, n. 2, p. 117-124, 2000.
HYLAND, P. W.; SOOSAY, C.; SLOAN, T. R. Continuous improvement and learning in the supply chain. International Journal of Physical Distribution \& Logistics Management, v. 33, n. 4, p. 316-335, 2003.

JABNOUN, N. Values underlying continuous improvement. The TQM Magazine, v. 13, n. 6, p. 381-387, 2001.

LEE, H. J. The role of competence-based trust and organizational identification in continuous improvement. Journal of Managerial Psychology, v. 19, n. 6, p. 623-639, 2004.

MARIN-GARCIA, J. A.; VAL, M. P.; MARTIN, T. B. Longitudinal study of the results of continuous improvement in an industrial company. Team Performance Management, v. 14, n. 1/2, p. 56-6, 2008.

MONTGOMERY, C. D.; RUNGER, G. C. Estatística aplicada e probabilidade para engenheiros. 2. ed. São Paulo: LTC, 2003.

MURRAY, P.; CHAPMAN, R. From continuous improvement to organizational learning: developmental theory. The Learning Organization, v. 10, n. 5, p. 272-282, 2003.

SAVOLAINEN, T. 1. Cycles of continuous improvement Realizing competitive advantages through quality. International Journal of Operations \& Production Management, v. 19, n. 11, p. 1203-1222, 1999.

TERZIOVSKI, M. Achieving performance excellence through an integrated strategy of radical innovation and continuous improvement. Measuring Business Excellence, v. 6, n. 2, p. 5-14, 2002.

TERZIOVSKY, M.; SOHAL, A. S. TQM in Australian manufacturing: factors critical to success. International Journal of Quality and Reliability Management, v. 17, n. 2, p. 158-167, 2000.

YOUSSEF, M.; ZAIRI, M. Benchmarking critical factors for TQM Part 11 - empirical results from different regions in the world. Benchmarking for Quality Management \& Technology, v. 2, n. 2, p. 3-19, 1995.

\title{
Critical factors for the continuous improvement in Brazilian manufacturing companies
}

\begin{abstract}
The aim of this paper is to identify and analyze critical factors in the development of continuous improvement $(\mathrm{Cl})$ activities in Brazilian manufacturing companies. A conceptual model of relationship between practices and results was tested through a survey conducted in $\mathbf{4 6}$ manufacturing companies. Factors such as: problems solving tools training, suggestion incentives, face-to-face communication, visits to the shop floor and adoption of incentive systems, have proved to be critical at reaching success in $\mathrm{Cl}$ activities. Through factorial analysis, two critical constructs concerning continuous improvement process were identified: 1) promotion of continuous improvement through incentive mechanisms; and 2) High level management support and leadership and management active involvement. It was observed that there is a statistically meaningful relationship between the use of problem solving techniques and the incentive mechanisms in face of the companies' performance.
\end{abstract}

\section{Keywords}

Continuous improvement. Critical factor. Factorial analysis. 